\title{
A life-threatening neonatal anomaly: congenital diaphragmatic hernia
}

\begin{abstract}
Congenital diaphragm hernias are cases result of mostly where there are in the diaphragm a defect. 'Bochdaleck 'type hernia is occurring commonly. Thought other types are rarely seen, common comprises diaphragmatic hernia, pulmonary hypoplasia, abdominal organs translocation into thorax. The other anomalies can associate. Diagnose might prenatal by using eggs. Polyhydramnios can warn. Mortality depend on usually account of organs translocation largely on degree on pulmonary hypoplasia and pulmonary hypertension. In treatment there are laparotomy, thoracotomy and minimally invasive methods.
\end{abstract}

Keywords: congenital, diaphragm, hernia, pulmonary hypoplasia, pulmonary hypertension
Volume 9 Issue I - 202 I

\author{
Hayriye Alp \\ GETAT(CAM), Necmettin Erbakan University Meram Medical \\ Faculty Hospital,Turkey
}

\begin{abstract}
Correspondence: Hayriye Alp, GETAT(CAM), Necmettin Erbakan University Meram Medical Faculty Hospital, Konya, Turkey, Email hayriyebal@yahoo.com
\end{abstract}

Received: July 06, 2020 | Published: February II, 202 |
Abbrevations: $\mathrm{CDH}$, congenital diaphragmatic hernia; CRP, c reactive protein; USG, ultra sonography; IV, intravenous

\section{Introduction}

While the incidence of congenital diaphragmatic hernia $(\mathrm{CDH})$ is $1 / 2500$ in live births, it is known to be $1 / 5000$ if still births are included. ${ }^{1} \mathrm{CDH}$ is $85-90 \%$ in left hemithorax, $10-15 \%$ in right hemithorax and $2 \%$ in bilateral. Approximately $60 \%$ of the cases are isolated and do not accompany any other anomaly to $\mathrm{CDH} .{ }^{2}$ In 1848 , Bochdaleck identified the hernia to be referred by his name. Other hernias: Bochdaleck may be parasternal or retrosternal. In 1940, Ledd and Gross suggested that surgical intervention should be performed within the first two days. ${ }^{2}$ With this case report, we opened a discussion to the fact that a 38-year-old mother was born at the maternity ward of our hospital with a normal birth, diagnosed with diaphragmatic hernia, and was discharged with healing on the 17th post-operative day. We wanted to examine the relationship between prenatal diagnosis possibilities, postnatal life and morbidity of newborns with diaphragmatic hernia followed and treated in our clinic.

\section{Case report}

There was no feature in the background of a 38 year old mother. The baby boy born with a normal birth had a birth weight of 3000 grams. The 1st minute (Min) postpartum Apgar score was 9 while the 5 th minute. When respiratory distress was noticed during normal physical examination, the baby was taken to the neonatal intensive care unit and followed up in the incubator. Despite giving free oxygen inside the incubator, junior's respiratory distress is increase was observed in the follow-up. Upward displacement of the abdominal organs was detected in the x-ray lung imaging of the baby in the case. The newborn specialist was diagnosed with congenital diaphragmatic hernia and pulmonary hypoplasia. The baby patient was intubated under sedation because his respiratory distress increased. The patient was administered intravenous (IV) $0.5 \mathrm{mcg}$ demizolam for sedation. 3.5 number intubation tubes were used in the intubation process of the baby. In order to prevent patient's strain, IV $6 * 0.5 \mathrm{mg}$ demizolam (midozalam) and IV $6 * 10 \mathrm{mcg}$ fentanyl (fentanyl citrate) were continued. Ampisina (ampicillin) IV $2 * 150 \mathrm{mg}$ and genta (gentamicin)
$1 * 12 \mathrm{mg}$ were given during follow-up in the neonatal intensive care unit. The patient was started with intravenous $240 \mathrm{cc} 10 \%$ dextrose infusion $8 * 2$ and total parenteral nutrition. Preparation was made for the operation of the patient who was consulted with pediatric surgery. Informed consent was obtained from the family. White blood cell $28.52 \mathrm{ul}$, hemoglobin $15.9 \mathrm{~g} / \mathrm{dl}$, platelet $304 \mathrm{ul}$. Arterial blood gas pH 7.28, pCO2 57.4, PO2 28.9, glucose 53, lactate dehydrogenase 445 were found in the examinations of the patient who was given ASA 3 risk. Before the operation was started, the operation room was heated with a radiant heater. The patient with umbilicus vascular catheter was monitored. Since it was operated as an intubation, $1 \%$ MAC sevoflurane was given for the maintenance of anesthesia. Due to the rapid metabolism of the drug, $3 \mathrm{mg}$ rocuronium, $3 \mathrm{mg}$ prednol, $5 \mathrm{mcg}$ fentanyl IV were used in total throughout the operation. The operation of the patient, whose vital signs remained stable throughout the operation, lasted for 22 minutes. Primary diaphragm repair was performed by performing bowel loops during the operation, and then thorax tube was inserted. Again, intubated transfer was provided to the intensive care unit with an incubator. During the intensive care period, 0.3 unit insulin was made after post-op 1st day blood sugar reached $420 \mathrm{mg} / \mathrm{dl}$ and returned to normal. During the postoperative care, the intubation tube that was displaced due to frequent straining reflex was renewed. During intensive care follow-up, a cuffless intubation tube was used with the recommendation of the newborn specialist. During the laboratory follow-up, antibiotic revision was made due to the $\mathrm{c}$ reactive protein (CRP) increase. The patient was extubated on the 5th postoperative day. On the 6th postoperative day, the patient's thorax tube was withdrawn and on the 17th day the patient was discharged.

\section{Discussion}

In the incidence studies, it is stated that approximately $58 \%$ of the cases belong to the male and $42 \%$ of the girls. (7) Our case was also a male gender. Defects in lung tissue during embryological development in the etiology of $\mathrm{CDH}$, phrenic nerve feeding disorder, development defect of diaphragmatic fibers in the herniation region, pleuroperitoneal canal defect, vitamin A deficiency, thalidomide, and anti-epileptics are accused. ${ }^{3,4}$ Prenatal diagnosis of $\mathrm{CDH}$ is possible with regular ultrasonography follow-ups. Detection of polyhydramnios in USG can be a stimulating finding for $\mathrm{CDH}$. It 
is stated that the prenatal diagnosis rate is $54 \%$ in the studies. ${ }^{5}$ The degree of pulmonary hypoplasia in $\mathrm{CDH}$ determines mortality. While the lungs of living fetuses are normal, bad kangaroo's values are indicative of poor prognosis. ${ }^{6}$ In experimental and clinical studies, they reported that the rate of pulmonary expansion (expiratory tidal volume / inspiratory pressure) may be important in determining lung hypoplasia and barotrauma susceptibility. ${ }^{7}$ Advances in the treatment of hypertension and treatment of cardiac pathologies significantly increase the survival in treatment. ${ }^{8}$ Since the pleura are damaged surgically during the operation, post-operative thorax tube application is common. In our case, a thorax tube was attached. However, Levison and Colvin stated that pneumothorax formation increases mortality in their studies. ${ }^{9,10}$ Thanks to the thorax tube, the fluid unit is naturally prevented in this area. The thorax tube placed on the hernia side may negatively affect the deflation of the lung by applying negative pressure. Since the hypoplastic lung is over-ventilated, pulmonary vascular resistance increases, which may lead to increased pulmonary damage. ${ }^{9} 5 .-6$ of gestation in the weeks, if the area of lung development is narrow, not enough bronchus and alveolar structure and number, can be reached; gas exchange is restricted as a result of increased distance in the interstitial space. ${ }^{11}$ In parallel, structural changes occur in the vascular structure, there is a marked thickening between the arteriolar muscle layers. Vasoconstriction is lowers blood flow, making the existing pathological condition worse. The degree of these changes is the most important factors affecting prognosis. ${ }^{11}$ The survival of children with $\mathrm{CDH}$ affects the accompanying anomalies, degree of anomaly and postpartum care..$^{11}$ Although $25 \%$ of these children show symptoms of hernia later in life, most continue their lives with normal lung function.

One of the complications is gastroesophageal reflux. In general, the surgical technique can be seen in patch repair cases and may be a cause of developmental delay. ${ }^{12}$ The cause of pulmonary complaints; It can be reflux, lung hypoplasia or ventilation sequels applied. If this condition becomes chronic as in premature infants, it needs longterm oxygen treatment. ${ }^{13}$ Other complications include decreased inspiratory muscle strength and minor airway obstructions. Chronic pulmonary hypertension requiring vasodilator treatment may be seen, albeit a little. ${ }^{14}$ In the study conducted by Rocha et al. ${ }^{14} 61$ congenital diaphragmatic hernia babies were examined and the average day of hospitalization was reported as 11 days in the neonatal intensive care unit. ${ }^{14}$ In our case, it was 15 days. The average age was found to be 4 days in the study. Our case was operated when she was 2 days old. In the same study, while the need for inotrope was $44 \%$, there was no need for inotrope drug in our case. ${ }^{14}$ New treatment options to reduce mortality were also investigated. In the 1960 s, Areechon and Reid stated that mortality was high due to lung hypoplasia at birth, and in 1976 they successfully applied extra corporal membrane oxygenation. ${ }^{15}$ The most feared risks of children with $\mathrm{CDH}$ in the long term are the development of neurological damage., ${ }^{8,12}$ An underlying brain injury increases this risk. Other complications that affect life in the long run are chest wall and skeletal deformities. ${ }^{8,12}$ In order to minimize and avoid such complications, early diagnosis and treatment options appropriate for the patient's clinic should be applied and the complication rate will decrease that much. Prenatal early diagnosis is very important in the treatment of the case. If the sociocultural level of the family is sufficient, the case can be noticed early and referred to an appropriate specialist. The case should be evaluated by pediatric surgery on time. It is vital that the patient be fully monitored during the transfer between the intensive care unit and the operating room.

\section{Result}

Extensive research is needed to increase the prenatal diagnosis rate and decrease mortality. Since it is a rare and followed disease, it should be studied for a long time. Informing families according to socioeconomic level is of great importance. Due to the fact that we are a child and maternity hospital, it is possible to encounter such rare cases frequently. The fact that the pediatric surgery team is technically competent and the presence of our neonatal care unit and neonatal specialist are factors that reduce morbidity in such risky cases. However, the fact that our hospital provides services to patients with Syrian origin is an extra challenge in terms of language.

\section{Acknowledgments}

None.

\section{Conflicts of interest}

The author declares that is no conflicts of interest.

\section{Funding}

None.

\section{References}

1. Deprest JA, Gratacos E, Nicolaides K, et al. Changing perspectives on the perinatal management of isolated congenital diaphragmatic hernia in Europe. Clin Perinatol. 2009;36(2):329-347.

2. Kline-Fath BM. Current advances in prenatal imaging of congenital diaphragmatic [corrected] hernia. Pediatr Radiol. 2012;42 Suppl 1:S74 S90.

3. Iritani I. Experimental study on embryogenesis of congenital diaphragmatic hernia. Anat Embryol (Berl). 1984;169(2):133-139.

4. Allan DW, Greer JJ. Pathogenesis of nitrofen-induced congenital diaphragmatic hernia in fetal rats. J Appl Physiol. 1997;83(2):338-347.

5. Gallot D, Boda C, Ughetto S, et al. Prenatal detection and outcome of congenital diaphragmatic hernia: a French registry-based study. Ultrasound Obstet Gynecol. 2007;29(3):276-283

6. Corbett HJ, Losty PD. Congenital diaphragmatic hernia. In Parikh DH, Crabbe DCG, Auldist AW, et al (editors): Pediatric thoracic surgery. Springer-Verlag London 2009, pp. 483-499.

7. Cloutier R, Fournier L, Major D. Index of pulmonary expansion: a new method to estimate lung hypoplasia in congenital diaphragmatic hernia. $J$ Pediatr Surg. 1992;27(4):456-458.

8. Frisk V, Jakobson LS, Unger S, et al. Long-term neurodevelopmental outcomes of congenital diaphragmatic hernia survivors not treated with extracorporeal membrane oxygenation. J Pediatr Surg. 2011;46(7):13091318.

9. Levison J, Halliday R, Holland AJA, et al. A population-based study of congenital diaphragmatic hernia outcome in New South Wales and the Australian capital territory, Australia, 1992-2001. J Pediatr Surg. 2006;41(6):1049-1053.

10. Colvin J, Bower C, Dickinson JE, et al. Outcomes of congenital diaphragmatic hernia: a population-based study in Western Australia. Pediatrics. 2005;116(3):e356-e363.

11. Pober BR. Genetic aspects of human congenital diaphragmatic hernia. Clin Genet. 2008;74(1):1-15.

12. Danzer E, Hedrick HL. Neurodevelopmental and neurofunctional 
outcomes in children with congenital diaphragmatic hernia. Early Hum Dev. 2011;87(9):625-632.

13. Taeng GY, Derry C, Marston L, et al. Reduction in ventilator-induced lung injury improves outcome in congenital diaphragmatic hernia? Pediatr Surg Int. 2008;24(2):145-150.
14. Rocha GM, Bianchi RF, Severo M, et al. Congenital diaphragmatic herniathe neonatal period (part I). Eur J Pediatr Surg. 2008; 18(4):219-223.

15. Waag KL, Loff S, Zahn K, et al. Congenital diaphragmatic hernia: a modern day approach. Semin Pediatr Surg. 2008;17(4):244-254. 